\title{
Retracting and seeking movements during laparoscopic goal-oriented movements. Is the shortest path length optimal?
}

\author{
Magdalena K. Chmarra Frank Willem Jansen • \\ Cornelis A. Grimbergen · Jenny Dankelman
}

Received: 13 March 2007/Accepted: 5 July 2007/Published online: 20 August 2007

(C) Springer Science+Business Media, LLC 2007

\begin{abstract}
Aims Minimally invasive surgery (MIS) requires a high degree of eye-hand coordination from the surgeon. To facilitate the learning process, objective assessment systems based on analysis of the instruments' motion are being developed. To investigate the influence of performance on motion characteristics, we examined goaloriented movements in a box trainer. In general, goal-oriented movements consist of a retracting and a seeking phase, and are, however, not performed via the shortest path length. Therefore, we hypothesized that the shortest path is not an optimal concept in MIS.

Methods Participants were divided into three groups (experts, residents, and novices). Each participant performed a number of one-hand positioning tasks in a box trainer. Movements of the instrument were recorded with the TrEndo tracking system. The movement from point $A$
\end{abstract}

M. K. Chmarra · C. A. Grimbergen · J. Dankelman Department of BioMechanical Engineering, Delft University of Technology, Mekelweg 2, 2628 CD, Delft, The Netherlands

F. W. Jansen

Department of Gynaecology, Leiden University Medical Center, Leiden, The Netherlands

C. A. Grimbergen

Department of Medical Physics, Academic Medical Center, Amsterdam, The Netherlands

M. K. Chmarra ( $\square)$

Department of BioMechanical Engineering, Faculty of

Mechanical, Maritime and Materials Engineering ( $3 \mathrm{mE})$,

Delft University of Technology, Mekelweg 2, 2628 CD, Delft,

The Netherlands

e-mail: m.k.chmarra@tudelft.nl to $B$ was divided into two phases: $A-M$ (retracting) and $M-B$ (seeking). Normalized path lengths (given in \%) of the two phases were compared.

Results Thirty eight participants contributed. For the retracting phase, we found no significant difference between experts [median (range) \%: 152 (129-178)], residents [164 (126-250)], and novices [168 (136-268)]. In the seeking phase, we find a significant difference $(<0.001)$ between experts [180 (172-247)], residents [201 (163287)], and novices [290 (244-469)]. Moreover, within each group, a significant difference between retracting and seeking phases was observed.

Conclusions Goal-oriented movements in MIS can be split into two phases: retracting and seeking. Novices are less effective than experts and residents in the seeking phase. Therefore, the seeking phase is characteristic of performance differences. Furthermore, the retracting phase is essential, because it improves safety by avoiding intermediate tissue contact. Therefore, the shortest path length, as presently used during the assessment of basic MIS skills, may be not a proper concept for analyzing optimal movements and, therefore, needs to be revised.

Keywords Minimally invasive surgery · Training · Motion analysis - Objective assessment .

Goal-oriented movement $\cdot$ Path length

Currently, there are various virtual-reality (VR) trainers available to train MIS skills [1,2]. These trainers have the advantage over box trainers of providing objective feedback about the performance of the user (performance parameters), which motivates residents to learn [3-5]. The most common parameter used to measure basic MIS skills objectively is task completion time. However, this parameter alone does 
not adequately evaluate the task performance [6]. Therefore, the time for completion is often used in conjunction with other parameters [1, 7-12]. One of the task-independent parameters that is used in each VR trainer is path length - the length of the curve described by the tip of the instrument over time [13]. Intuitively, a longer path length indicates a lessefficient movement and is often interpreted as lack of experience.

In MIS, goal-oriented movements (point-to-point movements) are very common (e.g., during grasping, placing a clip on the vessel, or while using diathermy). In general, a simple strategy to perform such a movement is to move along a straight line between an initial position and a specified target position [14, 15]. However, it is hardly possible to make such a movement during MIS. Movements of MIS instruments are done through the incision point, which acts as a pivot point. Therefore, point-to-point aiming movements in MIS may simply result in a curved workspace path (Fig. 1). Both these ways of performing point-to-point movements are very efficient. In general, however, goal-oriented movements are not performed via the shortest path; a pullback movement is performed before the specified target position is approached [16, 17]. Therefore, we hypothesized that the shortest path, as presently used to indicate more-efficient performance in MIS, is not a suitable reference.

The objective of this study was to examine goal-oriented movements during training of the basic MIS skills. The study consisted of two parts: first, we investigate whether goal-oriented movements can be split into two phases: a retracting phase and a seeking phase; and, subsequently, we investigate whether movements of the MIS instrument in these two phases depend on the surgeon's experience. The performance of the participants was analyzed and compared using normalized path lengths.

\section{Methods}

\section{Participants}

Experienced gynaecologists (with the experience of more than 100 MIS procedures), their residents (experience of 10-100 MIS procedures), and novices (medical students with no previous experience in MIS) were invited to participate in this study. Each participant, voluntarily enrolled in this study, was asked to complete a short questionnaire detailing demographic information and prior experience in laparoscopy.

\section{Task}

The hypothesis was tested using a simple one-hand positioning task, which requires touching the top of eight cylinders with the tip of the MIS instrument (Fig. 2). All cylinders were situated in a box trainer in various threedimensional (3D) positions. Every correctly touched cylinder resulted in the lighting of the lamp corresponding to this cylinder. The start/end point and the order of touching cylinders (indicated by the numbers located next to the cylinders) were the same for each participant.

To mimic the in vivo gynaecological MIS situation, all participants performed the task with their left hand, while the camera was held in the right hand. To provide the same conditions for each participant, the position of the task and the incision points for the camera and the MIS instrument were standardized in the box trainer. The image of a $0^{\circ}$ laparoscope was presented on a monitor.

Before the test, all participants were instructed how to perform the positioning task. Furthermore, they were allowed to make one trial before testing.

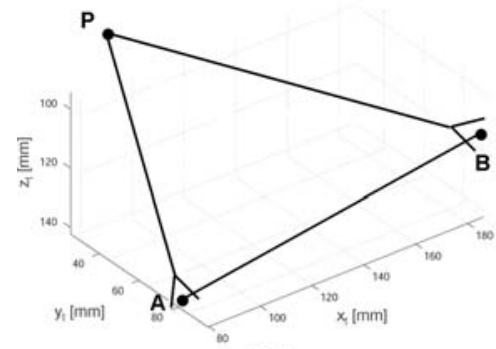

(a)

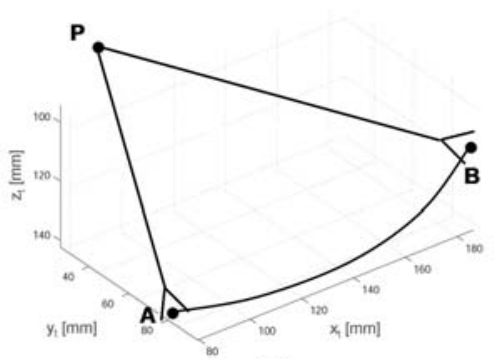

(b)

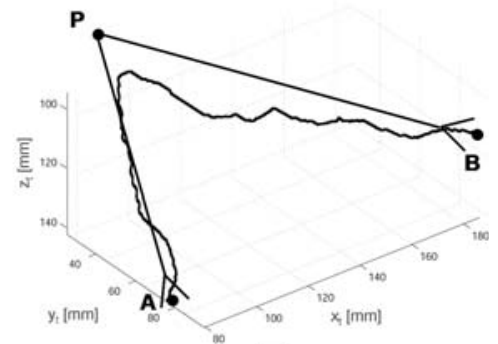

(c)

Fig. 1 Three strategies of performing a goal-oriented movement in MIS. (a) a movement along the straight line between the initial and the end position; (b) a movement along a curved path between the initial and the end position, which is the result of the movement of the MIS instrument through the incision point; and (c) a realistic movement performed by the surgeon during the goal-oriented task. A - the initial position; B - the end position; $\mathrm{P}$ - incision (pivoting) point 


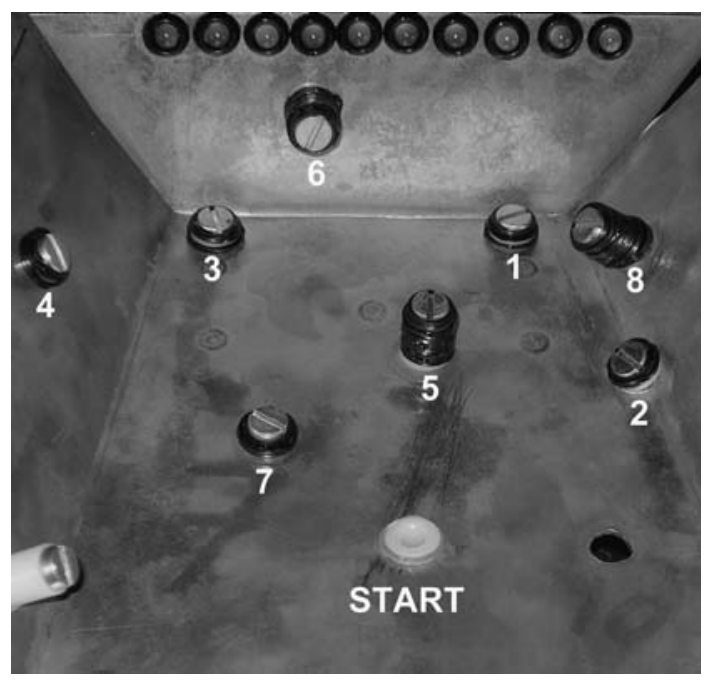

Fig. 2 The positioning task. The task requires touching the top of the eight cylinders (of varying 3D position) with the tip of the MIS instrument. A correctly touched cylinder results in lighting up a lamp (above on the picture) corresponding to this cylinder. The start/end point and the order of touching the cylinders are indicated by the numbers located next to the cylinders

\section{Data analysis}

Movements of the MIS instrument were recorded with our newly developed TrEndo tracking system with a sample frequency of $100 \mathrm{~Hz}$ [18]. The data of the eight simple goal-oriented movements performed by each participant during the task were analyzed. The first goal-oriented movement was defined by the movement between the start point and first cylinder. Each successive goal-oriented movement was defined by the movement between two consecutive cylinders (Fig. 2).

The retracting phase describes the pull-back movement of the MIS instrument in the direction of the pivoting point. Therefore, we analyzed the projected movements of the instrument in the $A B P$ plane (Fig. 3), which passes through the begin point of the movement (point $A$ ), the end point of the movement (point $B$ ), and the pivoting point (point $P$ ). To examine the deviation of the movement from the plane $A B P$, we also analyzed the movements projected in the plane $A B$, which is perpendicular to the plane $A B P$, and which passes through the points $A$ and $B$ (Fig. 3). Point $M$, defined as the point of the movement most distant from the line $A B$, was used to make a distinction between the retracting phase $A M$ (from point $A$ to point $M$ ) and the seeking phase $M B$ (from point $M$ to point $B$ ). The analysis of each of the two phases was done using a normalized path length, which was derived as follows:

$$
P L_{\text {normalized }}=\frac{P L_{\text {real }} \times 100}{P L_{0}}[\%]
$$

where $P L_{\text {real }}$ is the real path length (between $A$ and $M$ for retracting, and $M$ and $B$ for seeking phase), and $P L_{0}$ is the distance (length of the straight line) between $A$ and $M$ and $M$ and $B$ (for retracting, and seeking phases, respectively). The normalized path lengths of all eight successive goal-oriented movements were averaged per participant and analyzed.

\section{Statistics}

Data were analyzed using the Statistics Toolbox of MATLAB 7. Statistical analysis was performed using oneway analysis of variance (ANOVA), and Wilcoxon tests. A probability $p<0.05$ was considered to be statistically significant.

\section{Results}

Participants

Thirty-eight participants completed the task and the questionnaire. The group of experts consisted of 9 experts (age 38-59 years). The group of residents consisted of 17 gynaecological residents (age 29-41 years). The group of novices consisted of 12 medical students (age 23-28 years). All participants were right-handed.

\section{Retracting and seeking phases}

Typical trajectories for an expert and a novice performing the goal-oriented movement are presented in Fig. 4. The figure shows that the retracting and seeking phases can easily be distinguished from each other. No significant difference between the experts, residents, and novices was observed in the distance between point $M$ and the line $A B$.

Normalized path lengths of the two phases are presented in Fig. 5. In 3D space, the experts' normalized path length was shorter during the retracting phase [median (range) \%: 152 (129-178)] than during the seeking phase [180 (172247)]. Similarly, the residents' and novices' path lengths were shorter during the retracting phase [164 (126-250), and 168 (136-268)] than during the seeking phase [201 (163-287), and 290 (244-469), respectively].

In the $A B P$ plane, the experts' normalized path length in the retracting phase [132 (120-194)] was significantly shorter than in the seeking phase [199 (154-234)]. The normalized path lengths of residents and novices were also significantly shorter in the retracting phase [133 (108-492), and $180(118-287)]$ than in the seeking phase [208 (162$286)$, and 310 (252-469)]. 


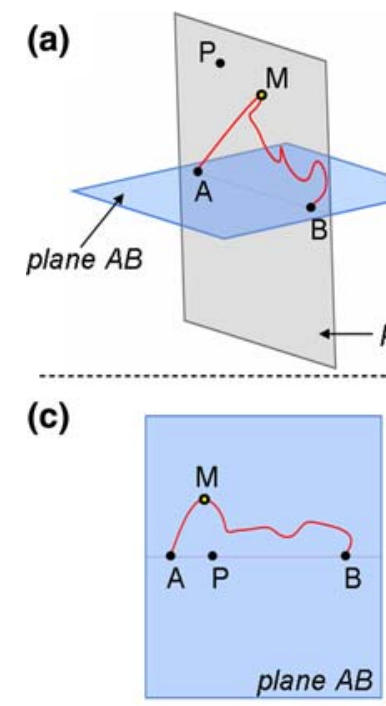

Fig. 3 The $A B P$ (described by the points $A, B$, and $P$ ) and $A B$ (that passes through points $A$ and $B$, and is perpendicular to the plane $A B P$ ) planes presented in an orthographic projection: (a) the $A B P$ and $A B$ planes, and the goal-oriented movement presented in the threedimensional space; (b) front view: projection of the goal-oriented movement in the $A B P$ plane; (c) top view: projection of the goal- (b)

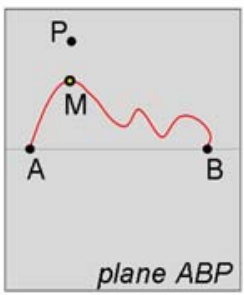

(d)

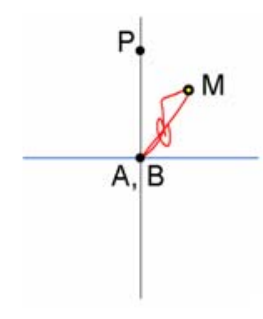

oriented movement in the $A B$ plane; and (d) side view: the $A B P$ and $A B$ planes, and the goal-oriented movement presented in a side view. $\mathrm{A}-$ the initial position; $\mathrm{B}-$ the end position; $\mathrm{M}-\mathrm{a}$ point, which is used to make a distinction between the retracting and the seeking phases; $\mathrm{P}$ - incision (pivoting) point
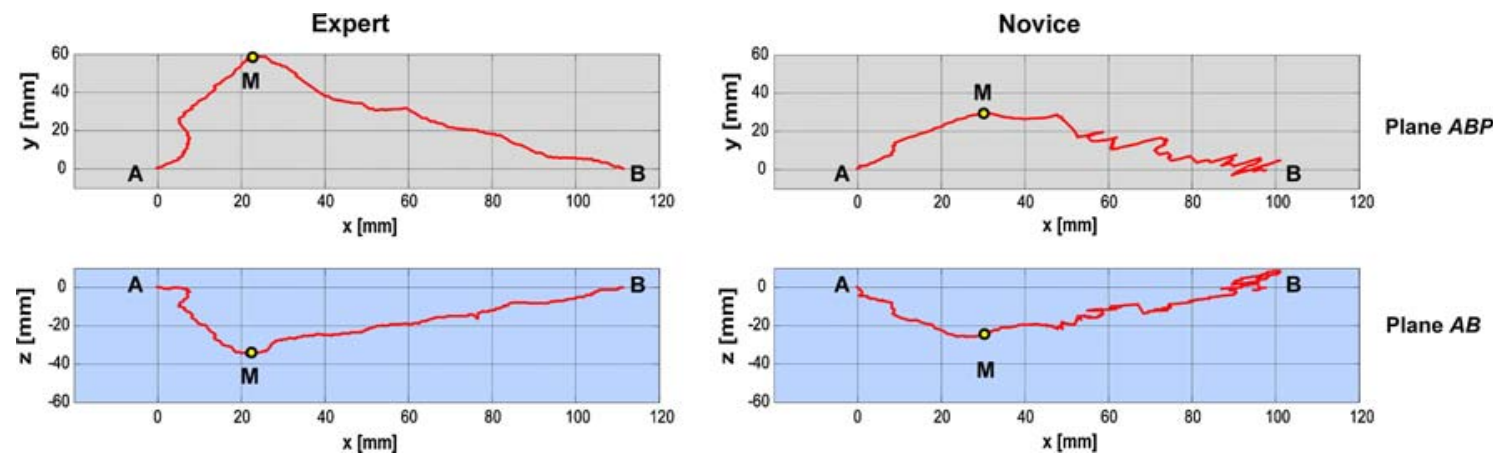

Fig. 4 The typical trajectories for an expert (left) and a novice (right) performing a goal-oriented movement. Top: projection of the goaloriented movement in the plane $A B P$. Bottom: projection of the goal-oriented movement in the plane $A B$. $\mathrm{A}-$ the initial position; $\mathrm{B}-$ the end position; $\mathrm{M}$ - a point, which is used to make a distinction between the retracting and the seeking phases

In the $A B$ plane, the experts' normalized path length was significantly shorter in the retracting phase [122 (100-200)] than in the seeking phase [183 (141-209)]. Normalized path lengths of residents and novices were also significantly shorter during the retracting phase [147 (107-264), and 207 (128-271)] than during the seeking phase [199 (162-285), and 310 (252-469)].

\section{Influence of the experience}

The experts', residents', and novices' normalized path lengths during the retracting and the seeking phases are presented in Fig. 6. The data in Fig. 6 are the same as the data in Fig. 5, but presented per phase for all groups. In 3D space, there was no significant difference between experts, residents, and novices for the retracting phase. The normalized path lengths of experts and residents were significantly shorter than the path length of the novices during the seeking phase. No significant difference between experts and residents was observed in that phase.

In the $A B P$ plane, we found no significant difference between experts, residents, and novices for the retracting phase. In the seeking phase, the normalized path lengths of experts and residents were significantly shorter than the 
Fig. 5 Normalized path lengths of the retracting and the seeking phases for experts, residents, and novices. Upper: normalized path length in 3D space.

Middle: normalized path length in the $A B P$ plane. Lower: normalized path length in the $A B$ plane. The results are presented as notched box-andwhisker plots, where every box has a line at every quartile, median, and upper quartile values. The whiskers are presented as lines that extend from each end of the box in order to show the extent of the rest of the data. The notches represent the $95 \%$ confidence interval for the median. Boxes whose notches do not overlap are significantly different $(p<0.05) . * * p<0.01$; $* * * p<0.001 ; \mathrm{E}-$ experts; $\mathrm{R}-$ residents; $\mathrm{N}$ - novices; 1 retracting phase; 2 - seeking phase
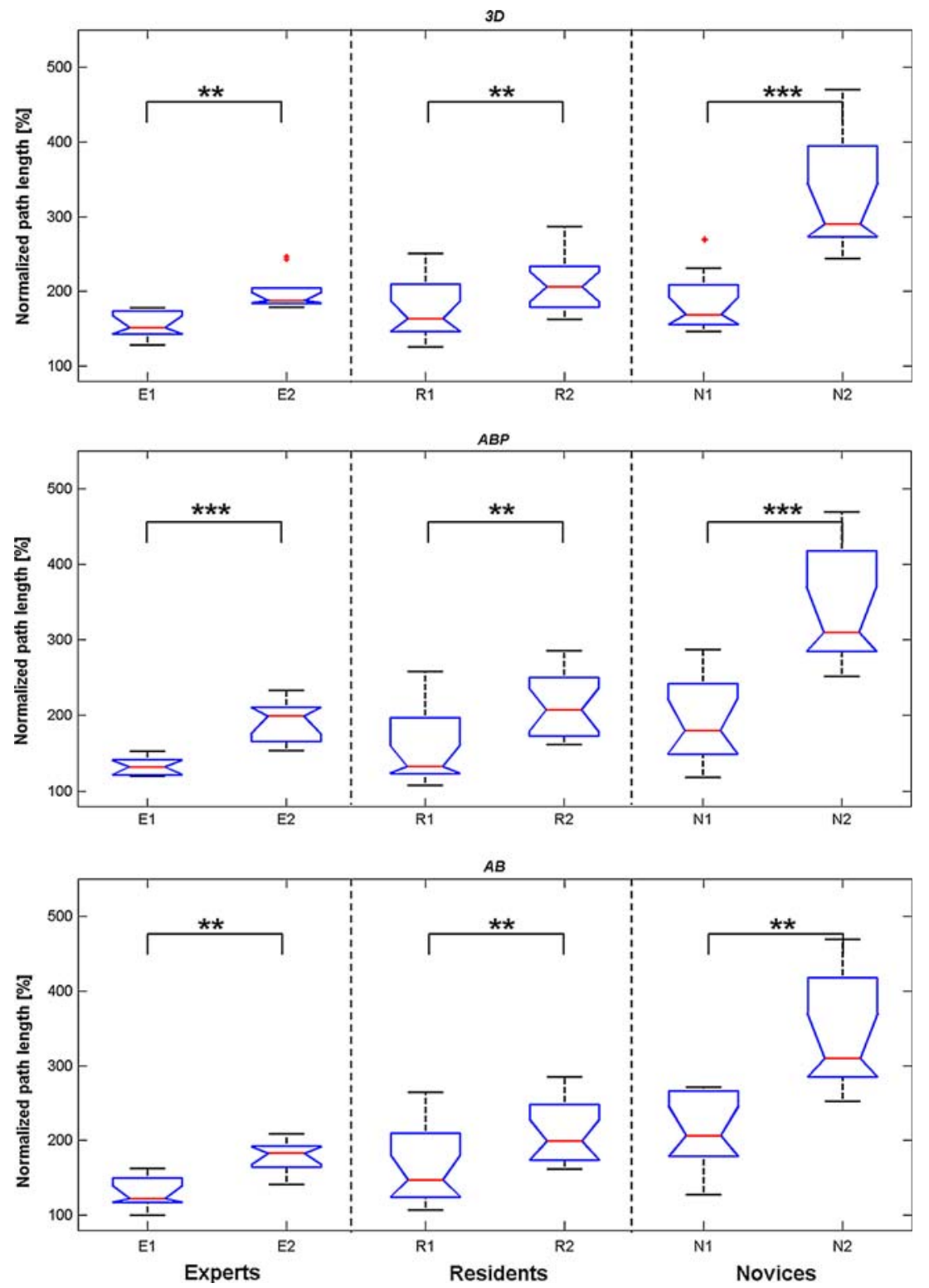

path length of novices. No significant difference between experts and residents was observed in that phase.

In the $A B$ plane, a significant difference between experts, residents, and novices was found for both phases. In both the retracting and the seeking phases, the normalized path lengths of the experts and residents were significantly shorter than the path length of the novices. No significant difference between experts and residents was observed for both phases.

\section{Discussion}

Our findings indicate that goal-oriented movements are not performed via the shortest path. The movements clearly distinguish a retracting and a seeking phase. The results show that the normalized path length during the retracting phase is significantly shorter than during the seeking phase. Furthermore, experience in MIS does only influence the seeking phase. Experts' and residents' normalized path lengths were significantly shorter than the path lengths of the novices. The shorter path length in the seeking phase implies better performance; therefore, the seeking phase is characteristic of the differences in performance. This finding confirms that the path length is an important measure in the assessment of basic MIS skills, in which the seeking phase is the only component that can distinguish between novice and expert.

The retracting phase is essential in MIS, because it improves safety of the patient by avoiding unpredicted 
Fig. 6 The influence of the experience on the performance of the retracting and the seeking phases. The data presented in this figure are the same as the data in Fig. 7, but presented per phase. ${ }^{*} p<0.05$; $* * * p<0.001$; $\mathrm{E}$ - experts; $\mathrm{R}$ - residents; $\mathrm{N}$ novices; 1 - retracting phase; 2 - seeking phase
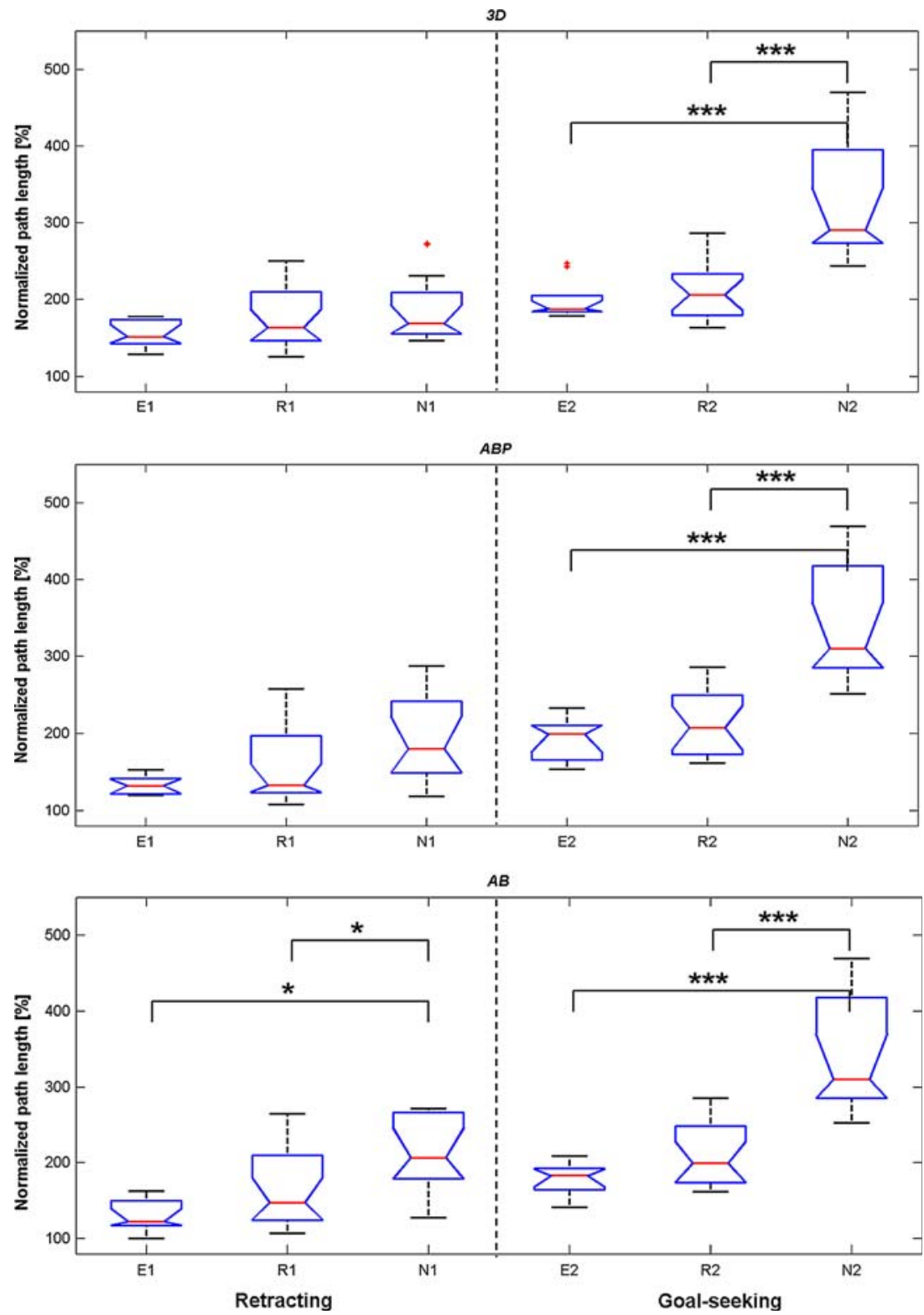

contact with the tissue. It seems that the best strategy to perform this phase safely is to pull back the instrument along its axis (in the direction of the pivoting point) and avoid any movements in the $A B$ plane, which can cause unpredicted contacts with the tissue. The results of this study confirm this assumption; experts' and residents' normalized path lengths are smaller in the $A B$ plane than the path length of the novices. Movements in the $A B$ plane can result in unintended tissue contact and be dangerous for the patient; therefore, it would be beneficial to let novices learn how to perform a more-precise retracting movement.

The experience in MIS does not influence the retracting phase in the $A B P$ plane. Moreover, a longer retraction of the MIS instrument in the direction of the pivoting point does not denote worse or less-efficient performance. In contrast, it may be a sign of the safer (for the patient) use of the MIS instruments. This finding is important, because it shows that the shorter path length, as presently used to indicate more-efficient performance in MIS, is not a correct concept for analyzing optimal movements in laparoscopy.

To investigate whether the retracting phase really represents safety, it is necessary to design a different study. For example, one possibility would be to include obstacles which, upon touching represent an error. With such a study design, it would be feasible to determine how much retraction is necessary and whether the experts have learnt the optimal retraction amount. The present study cannot be used to decide on these aspects.

The results of this study indicate that the comparison of the novices' path length to the experts' path length is an 
important and valid component of the overall criterionbased assessment of basic MIS skills. It is necessary to realize that this study was not designed to determine whether expert performance actually results in the optimal path length and, therefore, the results of this study cannot be used to indicate that experts' movements are optimal.

In the literature, there is a tendency to assess MIS performance using very basic terms (e.g., time, path length, penalty points), which do not show "the size and the nature of the gap between expert and novice performance" [13]. The results of this study show that the analysis of the separate phases of the movement in various planes can give such details. For example, movements of the MIS instrument along its axis (in and out the trocar) during the seeking phase characterize the surgeon's level of depth perception. Movements of the instrument in the $A B$ plane may indicate eye-hand coordination problems (e.g., orientation). Since every phase of the movement analyzed in different planes provides details about different limitations, it is crucial to analyze the movements in MIS separately for each phase and in both planes. Only then will the feedback about the performance and the nature of the limitations lead to goal-oriented training curricula.

This study shows for the first time the importance of proper analysis of the instruments' movements during training of MIS skills. The clinical impact of such extended analysis is that only in this way is it possible to implement a correct objective score that will measure and certify the competence of surgeons' basic psychomotor MIS skills in addition to the existing criteria for the assessment of MIS performance. Moreover, this extended motion analysis can result in improvement of the training of basic MIS skills, since it will identify the differences between the experts' and novices' performance, and which areas require more training.

\section{Conclusions}

Movements during laparoscopic tasks are not performed via the shortest path. Therefore, the shortest path length, as presently used for the assessment of basic MIS skills, may be not a proper concept for analyzing optimal movements and therefore needs to be revised. Goal-oriented movements should be split into two phases: a retracting and a seeking phase. Novices are less efficient in the seeking phase. This finding confirms that path length is an important measure in the assessment of basic MIS skills, but that the seeking phase is the only component that distinguishes between novice and expert. Furthermore, the retracting phase is very important in MIS, since it improves safety by avoiding intermediate tissue contact. Analyzing motions in the separate phases provides greater insight into the nature of the gap between expert and novice performance. Motion analysis in MIS should be seen as an addition to the existing criteria in assessing performance.

Acknowledgements The authors would like to thank the Medical Technology Development Department of the Academic Medical Center, Amsterdam for help in designing and manufacturing the TrEndo prototype. We also thank all gynaecologists, residents, and novices for participating in this study.

\section{References}

1. Halvorsen FH, Elle OJ, Fosse E (2005) Simulators in surgery. Minim Invasiv Ther 14:214-223

2. Schijven M, Jakimowicz J (2003) Virtual reality surgical laparoscopic simulators. How to choose. Surg Endosc 17:1943-1950

3. Aggarwal R, Moorthy K, Darzi A (2004) Laparoscopic skills training and assessment. Br J Surg 91:1549-1558

4. Grantcharov TP, Rosenberg J, Pahle E, Funch-Jensen P (2001) Virtual reality computer simulation. An objective method for the evaluation of laparoscopic surgical skills. Surg Endosc 15:242-244

5. Darzi A, Mackay S (2001) Assessment of surgical competence. Qual Health Care 10:64-69

6. Shah J, Buckley D, Frisby J, Darzi A (2003) Reaction time does not predict surgical skill. Br J Surg 90:1285-1286

7. Satava RM, Cuschieri A, Hamdorf J (2003) Metrics for objective assessment. Preliminary summary of the Surgical Skills Workshop. Surg Endosc 17:220-226

8. Cavallo F, Megali G, Sinigaglia S, Tonet O, Dario P (2006) A biomechanical analysis of surgeon's gesture in a laparoscopic virtual scenario. Stud Health Technol Inform MMVR 14:79-84

9. Hance J, Aggarwal R, Moorthy K, Munz Y, Undre S, Darzi A (2005) Assessment of psychomotor skills acquisition during laparoscopic cholecystectomy courses. Am J Surg 190:507-511

10. Grantcharov TP, Bardram L, Funch-Jensen P, Rosenberg J (2003) Learning curves and impact of previous operative experience on performance on a virtual reality simulator to test laparoscopic surgical skills. Am J Surg 185:146-149

11. Larsen CR, Grantcharov T, Aggarwal R, Tully A, Sorensen JL, Dalsgaard T, Ottesen B (2006) Objective assessment of gynaecologic laparoscopic skills using the LapSimGyn virtual reality simulator. Surg Endosc 20:1460-1466

12. Gallagher AG, Richie K, McClure N, McGuigan J (2001) Objective psychomotor skills assessment of experienced, junior, and novice laparoscopists with virtual reality. World J Surg 25:1478-1483

13. Cotin S, Stylopoulos N, Ottensmeyer M, Neumann P, Rattner D, Dawson S (2002) Metrics for laparoscopic skills trainers: The weakest link! Lect Notes Comput Sci 2488:35-43

14. Morasso P (1981) Spatial control of arm movements. Exp Brain Res 42:223-227

15. Flash T (1987) The control of hand equilibrium trajectories in multi-joint arm movements. Biol Cybern 57:257-274

16. Carrozzo M, McIntyre J, Zago M, Lacquaniti F (1999) Viewercentered and body-centered frames of reference in direct visuomotor transformations. Exp Brain Res 129:201-210

17. Reina GA, Moran DW, Schwartz AB (2001) On the relationship between joint angular velocity and motor cortical discharge during reaching. J Neurophysiol 85:2576-2589

18. Chmarra MK, Bakker NH, Grimbergen CA, Dankelman J (2006) TrEndo, a device for tracking minimally invasive surgical instruments in training set-ups. Sensor Actuat A-Phys 126:328334 